\title{
The Dinosaurs of the Early Jurassic Hanson Formation of the Central Transantarctic Mountains: Phylogenetic Review and Synthesis
}

\author{
N. D. Smith ${ }^{1,2}$, P. J. Makovicky ${ }^{1}$, D. Pol ${ }^{3}$, W. R. Hammer ${ }^{4}$, and P. J. Currie ${ }^{5}$ \\ ${ }^{1}$ Department of Geology, The Field Museum of Natural History, 1400 S. Lake Shore Drive, Chicago, IL 60605, USA (smithnd@uchicago.edu) and \\ (pmakovicky@fieldmuseum.org) \\ ${ }^{2}$ Committee on Evolutionary Biology, University of Chicago, 1025 E. $57^{\text {th }}$ Street, Culver 401, Chicago, IL 60637, USA \\ ${ }^{3}$ CONICET, Museo Paleontológico Egidio Feruglio, Av. Fontana 140, Trelew 9100, Chubut, Argentina (dpol@ mef.org.ar) \\ ${ }^{4}$ Department of Geology, Augustana College, $63938^{\text {th }}$ street, Rock Island, IL 61201, USA (WilliamHammer@augustana.edu) \\ ${ }^{5}$ Department of Biological Sciences, University of Alberta, CW 405 Biological Sciences Centre, Edmonton, Alberta, Canada \\ (philip.currie@ualberta.ca)
}

\begin{abstract}
The Hanson Formation of the Central Transantarctic Mountains has yielded a diverse Early Jurassic terrestrial fauna, which includes the nearly complete theropod dinosaur, Cryolophosaurus ellioti, and a fragmentary basal sauropodomorph dinosaur. The Hanson Formation dinosaurs are important for understanding early dinosaur evolution because: 1) they preserve a mosaic of morphological traits that render them useful for interpreting poorly known parts of the dinosaur evolutionary tree; 2) they are from the Early Jurassic, a critical period in early dinosaur evolution about which knowledge is scant; and 3) they are the only known Early Jurassic dinosaurs from Antarctica, making them particularly valuable for understanding patterns of biotic interchange during this time. Recent research suggests that Cryolophosaurus belongs to a geographically widespread clade of mid-sized, Early Jurassic theropods with cranial crests that includes Dilophosaurus wetherilli, 'Dilophosaurus' sinensis, and Dracovenator, and renders Coelophysoidea sensu lato non-monophyletic. The Antarctic sauropodomorph represents a distinct taxon that is a member of a similarly diverse massospondylid clade. This taxon shares a number of features with more derived sauropodomorphs, and provides additional evidence for the paraphyly of Prosauropoda. The phylogenetic relationships of the Antarctic dinosaurs are also consistent with a pattern of worldwide faunal homogeneity between Early Jurassic continental biotas. Furthermore, these analyses support a "ladder-like" arrangement for basal theropod and basal sauropodomorph phylogeny, suggesting that these groups passed through "coelophysoid" and "prosauropod" stages of morphological organization early in their respective evolutionary histories.
\end{abstract}

Citation: Smith, N. D., P. J. Makovicky, D. Pol, W. R. Hammer, and P. J. Currie (2007), The dinosaurs of the Early Jurassic Hanson Formation of the Central Transantarctic Mountains: Phylogenetic review and synthesis, in Antarctica: A Keystone in a Changing World - Online Proceedings of the $10^{\text {th }}$ ISAES, edited by A. K. Cooper and C. R. Raymond et al., USGS Open-File Report 2007-1047, Short Research Paper 003, 5 p.; doi:10.3133/of2007-1047.srp003.

\section{Introduction}

The Hanson Formation consists of siliceous siltstones, tuffs, and tuffaceous sandstones, and outcrops extensively on several peaks in the Beardmore Glacier region of the Central Transantarctic Mountains (Elliot, 1996). It was formally named in 1996 to differentiate its tuffaceous layers from the underlying, volcanoclastic-poor Falla Formation. Over the course of two field seasons of collecting (1990-91, 2003-04), a quarry near the base of the Hanson Formation at approximately 4,100 meters on Mt. Kirkpatrick has yielded a diverse Early Jurassic vertebrate fauna (Hammer and Hickerson, 1994; Smith et al., in press; Hammer and Smith, in review; Smith and Pol, in review). Faunal elements include a nearly complete skeleton of the theropod dinosaur Cryolophosaurus ellioti, a distal left femur and articulated right metatarsus of a basal sauropodomorph dinosaur (Fig. 1), a single postcanine tooth from the right maxilla of a tritylodont, and a pterosaur humerus. The phylogenetic relationships of these taxa have remained enigmatic, though recent work (Smith et al., in press; Smith and Pol, in review) summarized here is shedding new light on the relationships of these animals and character evolution within their respective groups. These studies also provide critical preliminary information on biogeographic patterns involving Antarctica during the Early Jurassic, for which data were previously lacking.

\section{Phylogenetic Review}

\section{Cryolophosaurus ellioti}

Hammer and Hickerson (1994) originally noted the interesting mosaic of features present in Cryolophosaurus, and though they provided no detailed assessment of the taxon's relationships, they did suggest possible affinities with Middle-Late Jurassic tetanurans. Cryolophosaurus posseses a number of plesiomorphic characteristics with respect to tetanuran theropods, concentrated primarily in the post-cranial skeleton (e.g., amphicoelous cervical centra, a sigmoidal femur with an anteromedially directed head, a low, triangular astragalar ascending process; Smith et al., in press). In contrast, several putatively derived features are present in the skull (e.g., the antorbital fossa extends onto the lateroventral side of the nasal, the presence of nasolacrimal crests, an expanded anterior end of the jugal, a deep surangular, and a pendant medial process on the articular; Smith et al., in press). This interesting combination of morphological characters, coupled with the current state of flux in basal theropod relationships, has likely contributed to the lability of Cryolophosaurus in recent phylogenetic 


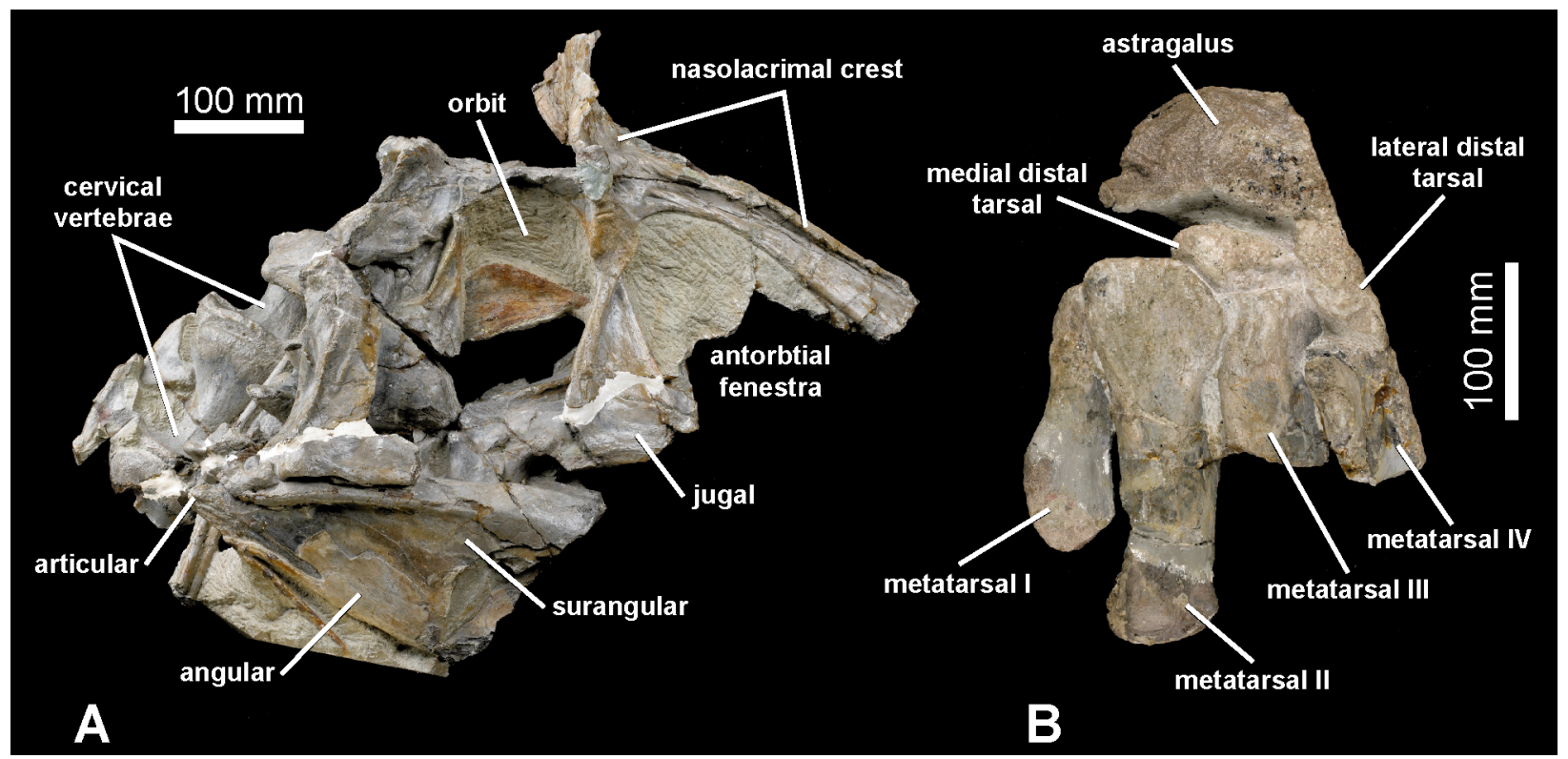

Figure 1. Skull of Cryolophosaurus ellioti (FMNH PR1821) in right lateral aspect (A). Right astragalus, distal tarsals, and metatarsals I-IV of the Antarctic sauropodomorph (FMNH PR1823) in posterior aspect (B). Photographs by J. Weinstein (FMNH).

analyses, which have recovered this taxon as an allosauroid (Sereno et al., 1996), or as the basal-most tetanuran (Carrano and Sampson, 2003; Smith et al., 2005). A recent in-depth morphological description of Cryolophosaurus and comprehensive phylogenetic analysis of basal theropod dinosaurs (Smith et al., in press) suggests that the traditional content of Coelophysoidea is non-monophyletic, and that Cryolophosaurus forms a clade with some traditional 'coelophysoids' (Dilophosaurus wetherilli), as well as some newly described and poorly known Early Jurassic theropods (Dracovenator regenti Yates, 2005; and 'Dilophosaurus' sinensis Hu, 1993). This clade of medium-bodied, Early Jurassic theropods is more closely related to a (Neoceratosauria + Tetanurae) clade than to the more basal 'coelophysoids' (i.e., Liliensternus + Coelophysidae; Fig. 2). Although support for relationships in this part of the tree is not robust, it appears that the mosaic of morphological features present in these poorly known Early Jurassic taxa is driving the phylogenetic signal. Many of these taxa possess classic 'coelophysoid' features (e.g., a low angle between the alveolar and anterior margin of the premaxilla, a subnarial gap, a raised ventral margin of the antorbital fossa, etc.), but also share features with more derived tetanurans and/or neoceratosaurs (e.g., keyhole-shaped orbit, anteriorly positioned maxillary tooth row, lacrimal fenestra, deep surangular, etc.). The effects of missing data on character optimization confound the identification of unambiguous synapomorphies at the base of the ('Dilophosaurus' sinensis, Dracovenator, Dilophosaurus wetherilli, Cryolophosaurus) clade, though several features that may support this grouping include: the contribution of the posterodorsal process of the premaxilla to a blade-like nasal crest, a foramen at the base of the nasal process of the premaxilla, extension of the antorbital fossa onto the nasal, and the presence of a nasolacrimal crest (Smith et al., in press).

\section{Hanson Formation Sauropodomorph}

Hammer and Hickerson (1996) tentatively referred the fragmentary remains of a basal sauropodomorph from the Hanson Formation to the family Plateosauridae, though this referral was not based on any detailed anatomical features beyond the relative size of the material. As with basal theropod systematics, the taxonomy and phylogenetic relationships of basal sauropodomorph dinosaurs are experiencing considerable revision, with the traditional grouping of basal members within a monophyletic Prosauropoda supported or rejected to varying degrees (Galton and Upchurch, 2004; Yates, 2006, 2007; Smith and Pol, in review; see also contributions in Barrett and Batten, 2007). Our research has revealed that the Antarctic material represents a new taxon, clearly distinguished from Plateosaurus and other basal sauropodomorphs by several autapomorphic features in the metatarsus (Smith and Pol, in review). A preliminary phylogenetic analysis modified from the comprehensive dataset of Yates $(2006,2007)$ suggests that the new Antarctic taxon is allied with the massospondylid (sensu Yates, 2006) taxa Massospondylus, Coloradisaurus, and Lufengosaurus (Smith and Pol, in review; Fig. 2). Several synapomorphies shared by the Antarctic sauropodomorph and one or more of these massospondylids include: the presence of a proximolateral flange on the plantar surface of metatarsal II, a well-developed facet on metatarsal II for articulation with the medial distal tarsal, and a 


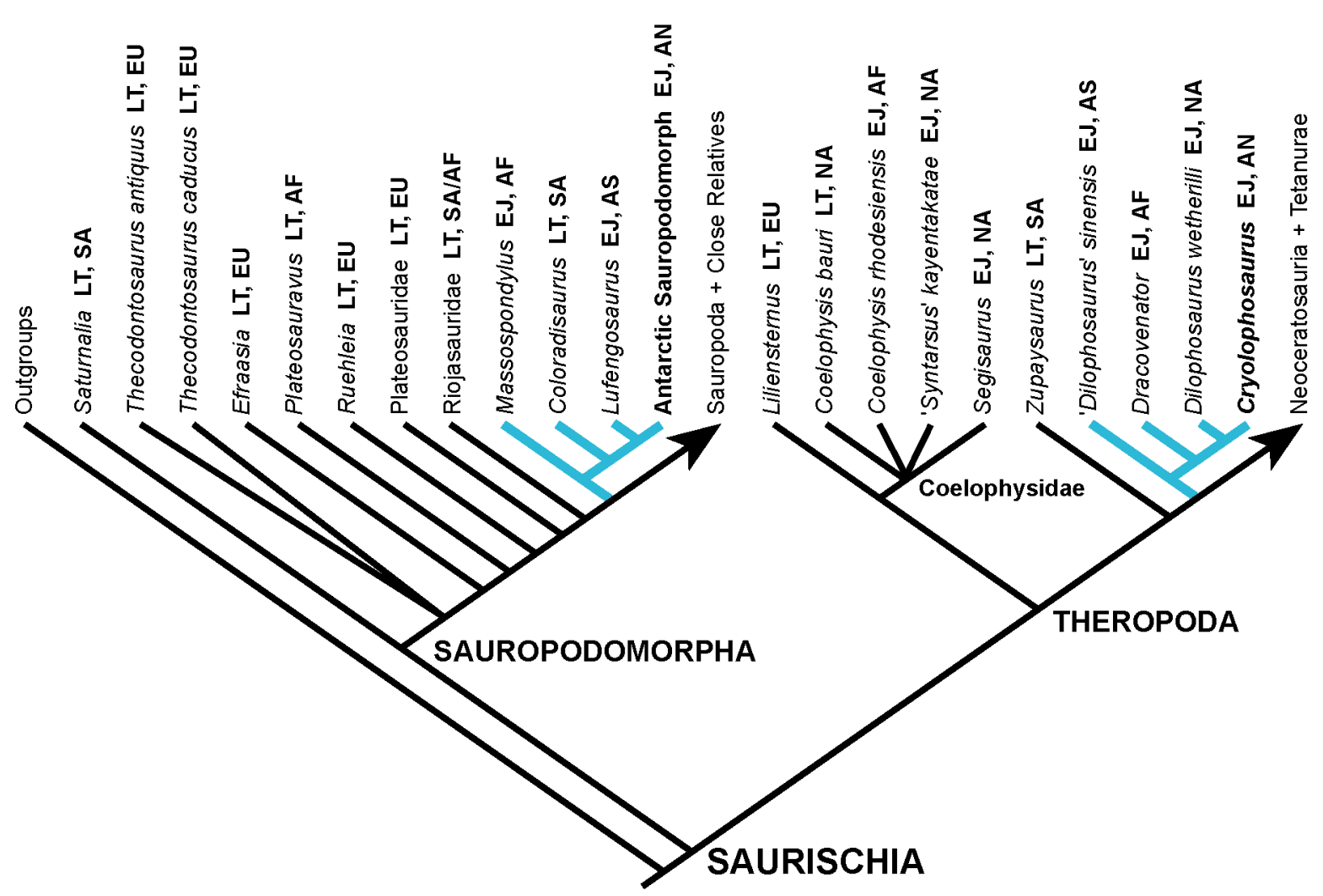

Figure 2. Generalized phylogeny of saurischian dinosaurs pruned to display basal relationships. Sauropodomorpha phylogeny is taken from Smith and Pol (in review), and Theropoda phylogeny is taken from Smith et al. (in press). The Massospondylidae clade, which includes the Antarctic sauropodomorph, and the medium-bodied, Early Jurassic theropod clade, which includes Cryolophosaurus, are both represented by thickened, colored branches. General temporal and geographic distributions are listed after each taxon name. Abbreviations: AF, Africa; AN, Antarctica; AS, Asia; EJ, Early Jurassic; EU, Europe; LT, Late Triassic; NA, North America; SA, South America.

subtrapezoidal proximal outline of metatarsal III (Smith and Pol, in review). The results of these recent analyses of basal sauropodomorph phylogeny suggest that it is the mosaic of plesiomorphic and derived features present in these taxa that are contributing to the breakdown of a traditional, and more inclusive 'prosauropod' clade (Yates, 2006, 2007; Smith and Pol, in review).

\section{Discussion}

\section{Paleobiogeographic Implications}

The role of Antarctica in the paleobiogeography of terrestrial vertebrates during the Early Jurassic has remained essentially unknown. Detailed anatomical description and phylogenetic analysis of taxa from the Hanson Formation fauna can allow for the first time a critical assessment (albeit preliminary and tentative), of paleobiogeographic patterns involving Antarctica. Extreme faunal homogeneity has been hypothesized for Early Jurassic continental biotas based on cluster analysis of faunal lists (Shubin and Sues, 1991), and the lack of statistical support for any hierarchical pattern of continental area relationships in the Early Jurassic is at least consistent with (though cannot be taken as positive evidence of), such a pattern (Upchurch et al., 2002). The phylogenetic relationships recovered by Smith et al. (in press), and Smith and Pol (in review) for basal saurischian dinosaurs is consistent with a pattern of Early Jurassic faunal homogeneity in two important ways. At a very general level, hierarchical patterns of continental area relationships implied by the phylogeny of the Early Jurassic medium-bodied theropod clade and the Early Jurassic members of the massospondylid clade are inconsistent with each other (Fig. 3). Specifically, the pattern within massopondylids would suggest closer faunal affinities between the Hanson Formation and the Lower Lufeng Formation of China than with the Upper Elliot Formation of southern Africa, while the pattern recovered for basal theropods implies the Hanson Formation fauna is more similar to that of the Kayenta Formation of North America or the Upper Elliot Formation. Perhaps more convincing is that multiple taxa from within several Early Jurassic faunas were included in the phylogenetic analyses of Smith et al. (in press), and Smith and Pol (in review), and were not recovered as closely related. For example, both Dilophosaurus wetherilli and 'Syntarsus' kayentakatae are known from the Kayenta Formation of the southwestern United States, but are distantly related to each other (Smith et al., in press). Likewise, Dracovenator and Coelophysis 


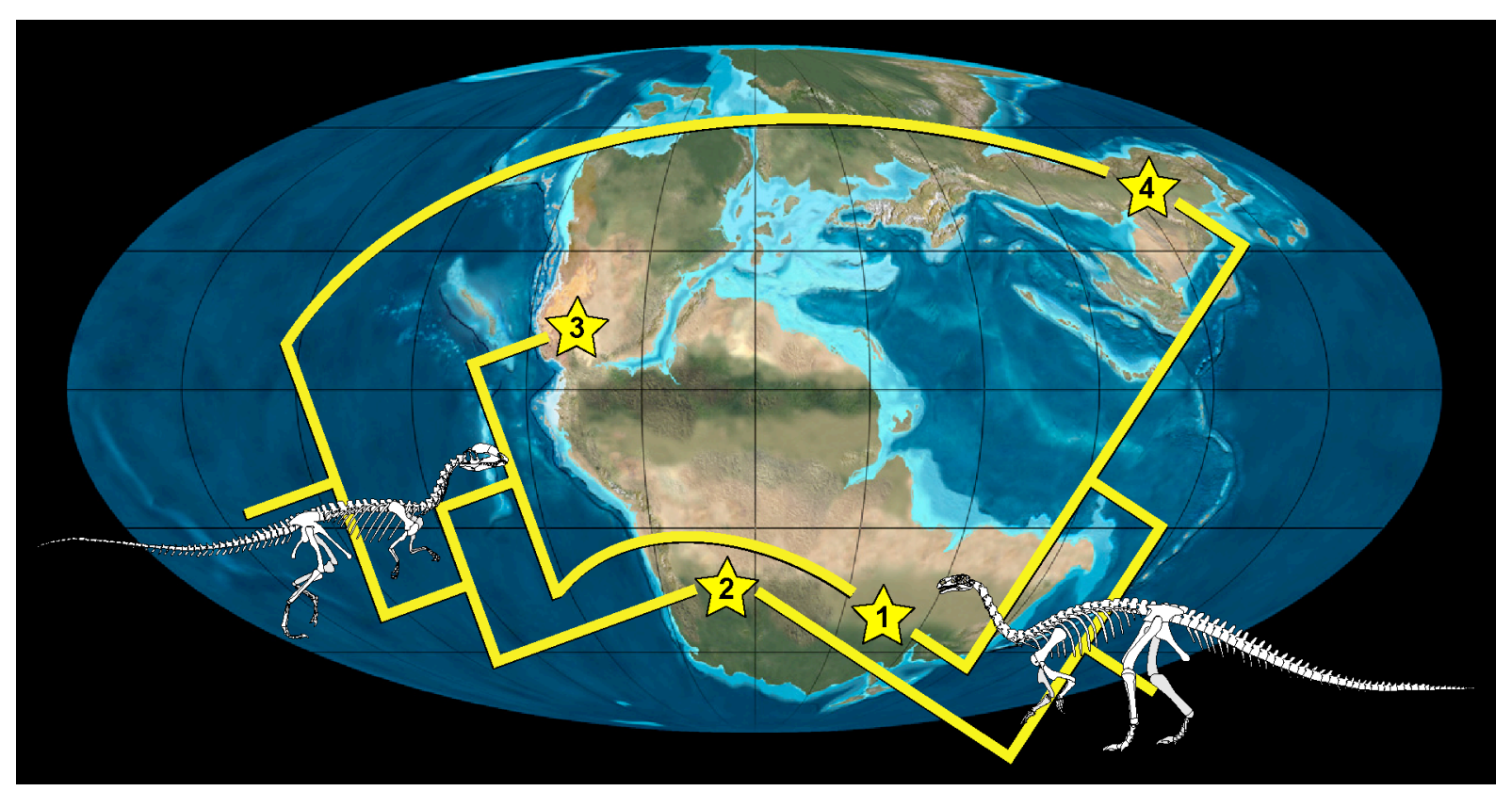

Figure 3. Phylogenetic relationships of the Early Jurassic members of the massospondylid sauropodomorph clade (right), and the medium-bodied, Early Jurassic theropod clade (left), mapped onto an Early Jurassic paleogeographic reconstruction. Labeled faunas: (1) Hanson Formation, Antarctica; (2) Massospondylus Range Zone, Southern Africa; (3) Kayenta Formation, Southwestern United States; (4) Lower Lufeng Formation, China. Paleogeographic map from Blakey (2006: http://jan.ucc.nau.edu/). Skeletal art courtesy of C. Brochu, and copyright of Tim Rowe (1993).

rhodesiensis are not close relatives, even though both taxa are from formations within the Massospondylus RangeZone of southern Africa (Smith et al., in press). A variety of basal sauropodomorphs are also known from the Lower Lufeng Formation, including Lufengosaurus, Jingshanosaurus, and Yunnanosaurus, yet they are not more closely related to each other than they are to taxa from other continental faunas (Yates, 2006, 2007; Smith and Pol, in review). A lack of endemism within the Lower Lufeng fauna is interesting given the geographic distinctiveness of this Early Jurassic fauna (Fig. 3). Furthermore, massospondylids may also be present in the Kayenta Formation (USA), and the Cañon del Colorado Formation of Argentina (Galton and Upchurch, 2004), suggesting they are a widespread and diverse clade.

\section{Paleobiological Implications}

The recovery of traditional Coelophysoidea and Prosauropoda clades as non-monophyletic, and the asymmetric topologies recovered for basal theropod and basal sauropodomorph phylogeny, implies these groups passed through "coelophysoid" and "prosauropod" stages of morphological organization early in their histories. These generalized body forms, coupled with poorly constrained saurischian outgroups and limited sampling of Early Jurassic taxa possessing transitional morphologies, has likely contributed to the difficulty in inferring basal relationships within Theropoda and Sauropodomorpha.

The temporal occurrences and relationships of basal sauropodomorphs suggest that many Early Jurassic taxa are relics of lineages that diversified in the Late Triassic
(Yates, 2006). Though the clade clearly originated in the Norian (Late Triassic), the Massospondylidae likely represent a secondary radiation of basal sauropodomorphs in the Early Jurassic. Currently, there is not enough resolution within Coelophysidae to determine whether its Early Jurassic members represent similarly diverse relics from Late Triassic lineages, or a temporally restricted Early Jurassic radiation (Smith et al., in press). However, the medium-bodied theropod clade that includes Cryolophosaurus appears to represent a secondary Early Jurassic radiation of basal theropods, similar to the Massospondylidae in geographic and taxonomic diversity. Even accepting traditional hypotheses of basal theropod and basal sauropodomorph phylogeny, it is clear that multiple lineages of both groups crossed the TriassicJurassic boundary. The footprint record of North America suggests that abundance patterns may have differed across the boundary, however (Olsen et al., 2002). Determining the effects, if any, the Late Triassic mass extinction had on the diversification of these early saurischians will require increased taxonomic sampling and a more solid understanding of group relationships.

Though none of the basal theropods included in the study of Smith et al. (in press) form endemic clades, there is a weak phylogenetic signal for body size, with Cryolophosaurus, Dilophosaurus wetherilli, Dracovenator, and 'Dilophosaurus' sinensis all estimated to be between 5.5 and 6.5 meters in body length. The increase in body-size observed in these Early Jurassic taxa is consistent with the dramatic appearance of correspondingly sized footprints immediately above the 
Triassic-Jurassic boundary (Olsen et al., 2002). Several small-bodied coelophysids, including Coelophysis bauri, Coelophysis rhodesiensis, 'Sytnarsus' kayentakatae, and Segisaurus, also form a monophyletic group. Thus, in a few Early Jurassic faunas it appears that several unrelated basal theropods co-existed in sympatry, with some evidence for possible resource partitioning provided by differences in body size. In addition to differences in body-size, nearly all Late Triassic and Early Jurassic theropods possess some form of cranial crest, which differ markedly from each other in construction and form. Cryolophosaurus is well-known for its unique transversely expanded and furrowed crest (Fig. 1), but the increased taxonomic sampling and recovered phylogeny of Smith et al. (in press) suggests that cranial ornamentation in basal theropods was more ubiquitous that previously thought. Because none of these structures likely served a combative function, and no convincing evidence yet exists for dimorphism (due mainly to a lack of sufficient sample size), species recognition represents a particularly intriguing explanation for the diversity of cranial crests in basal theropod dinosaurs (Padian et al., 2004). This inference is supported by the general pattern of geographically undifferentiated faunas in the Late Triassic and Early Jurassic, and by the probable sympatry of basal theropods in several well-known Early Jurassic faunas (Attridge et al., 1985; Shubin et al., 1991; Shubin and Sues, 1991; Sues and Reisz, 1995; Irmis, 2004).

\section{Summary}

Recent research on the dinosaurs of the Early Jurassic Hanson Formation of the central Transantarctic Mountains is summarized here. Cryolophosaurus ellioti belongs to a geographically widespread clade of mediumbodied, Early Jurassic theropods, while the Antarctic sauropodomorph represents a new taxon that is a member of the similarly diverse and widespread Massospondylidae. The phylogenetic relationships of the Antarctic dinosaurs are consistent with a pattern of extreme faunal homogeneity between Early Jurassic continental biotas. Furthermore, these analyses support a "ladder-like" arrangement for basal theropod and basal sauropodomorph phylogeny, suggesting that these groups passed through "coelophysoid" and "prosauropod" stages of morphological organization early in their respective evolutionary histories. Future exploration and collection in the Hanson Formation and underlying Falla Formation will be critical to testing phylogenetic and biogeographic patterns involving the Antarctic dinosaurs, and the Antarctic fauna as a whole.

\footnotetext{
Acknowledgements

We would like to thank the proceedings editors as well as the organizers and participants of ISAES X. We also thank S. J. Nesbitt and A. H. Turner for insightful reviews of the manuscript. Science and support staff based in McMurdo Station, Antarctica also deserve thanks for making this research possible. Financial support for the work discussed in this contribution has been provided by grants from the Jurassic Foundation, the Welles Fund (UCMP), the Wilkins Fund (U. of Iowa), and the University of Iowa Student Government to N. D. Smith,
}

NSF grant EAR-0228607 to P. J. Makovicky, NSF grant OPP-0229698 to W. R. Hammer, and grants from the Theodore Roosevelt Fund (AMNH), and the Annette Kade Fund to D. Pol.

\section{References}

Attridge, J., A. W. Crompton, and F. A. Jenkins, Jr. (1985), The southern Africa Liassic prosaurupod Massospondylus discovered in North America, J. Vert. Pal., 5, 128-132.

Barrett, P. M., and D. J. Batten (2007), Evolution and palaeobiology of early sauropodomorph dinosaurs, Special Papers in Palaeontology, 77.

Carrano, M., and S. D. Sampson (2003), The evolutionary history of basal theropod dinosaurs, J. Vert. Pal., 23(suppl. 3), 38-39A.

Elliot, D. H. (1996), The Hanson Formation: a new stratigraphical unit in the Transantarctic Mountains, Antarctica, Antarctic Science, 8(4), 389-394.

Galton, P. M., and P. Upchurch (2004), Prosauropoda, in The Dinosauria, second edition, edited by D. B. Weishampel, P. Dodson, and H. Osmólska, pp. 232-258, University of California Press, Berkeley.

Hammer, W. R., and W. J. Hickerson (1994), A crested theropod dinosaur from Antarctica, Science, 264, 828-830.

Hammer, W. R., and W. J. Hickerson (1996), Implications of an Early Jurassic vertebrate fauna from Antarctica, in The Continental Jurassic, edited by M. Morales, pp. 215-218, Museum of Northern Arizona Bulletin, 60.

Hammer, W. R., and N. D. Smith (in review), A tritylodont postcanine from the Hanson Formation of Antarctica, J. Vert. Pal.

Hu, S. (1993), A new Theropoda (Dilophosaurus sinensis sp. nov.) from Yunnan, China, Vert. PalAs., 31(1), 65-69.

Irmis, R. B. (2004), First report of Megapnosaurus (Theropoda: Coelophysoidea) from China, PaleoBios, 24, 11-18.

Olsen, P. E., D. V. Kent, H.-D. Sues, C. Koeberl, H. Huber, A. Montanari, E. C. Rainforth, S. J. Fowell, M. J. Szajna, and B. W. Hartline (2002), Ascent of dinosaurs linked to an iridium anomaly at the Triassic-Jurassic Boundary, Science, 265, 1305-1307.

Padian, K. P., J. Horner, and J. Dhaliwal (2004), Species recognition as the principal cause of bizarre structures in dinosaurs, J. Vert. Pal. 24(suppl. 3), 100A.

Shubin, N. H., A. W. Crompton, H. -D. Sues, and P. E. Olsen (1991), New fossil evidence on the sister-group of mammals and early Mesozoic faunal distributions, Science, 251, 1063-1065.

Shubin, N. H., and H. -D. Sues (1991), Biogeography of early Mesozoic continental tetrapods: patterns and implications, Paleobiology, 17(3), 214-230.

Smith, N. D., W. R. Hammer, and P. J. Currie (2005), Osteology and phylogenetic relationships of Cryolophosaurus ellioti (Dinosauria: Theropoda): Implications for basal theropod evolution, J. Vert. Pal., 25(suppl. 3), 116-117A.

Smith, N. D., P. J. Makovicky, W. R. Hammer, and P. J. Currie (in press), Osteology of Cryolophosaurus ellioti (Dinosauria: Theropoda) from the Early Jurassic of Antarctica and implications for early theropod evolution, Zool. J. Linn. Soc.

Smith, N. D., and D. Pol (in review), Anatomy of a basal sauropodomorph dinosaur from the Early Jurassic Hanson Formation of Antarctica, Acta Pal. Pol.

Sues, H. -D., and R. R. Reisz (1995), First record of the early Mesozoic sphenodontian Clevosaurus (Lepidosauria: Rhnchocephalia) from the southern hemisphere, J. Vert. Pal., 69, 123-126.

Upchurch, P., C. A. Hunn, and D. B. Norman (2002), An analysis of dinosaurian biogeography: evidence for the existence of vicariance and dispersal patterns caused by geological events, Proc. R. Soc. Lond. B, 269, 613-621.

Yates, A. M. (2005), A new theropod dinosaur from the Early Jurassic of South Africa and its implications for the early evolution of theropods, Palaeont. Afr., 41, 105-122.

Yates, A. M. (2006), Solving a dinosaurian puzzle: the identity of Aliwalia rex Galton, Hist. Biol., 4, 1-31.

Yates, A. M. (2007), The first complete skull of the Triassic dinosaur Melanorosaurus Haughton (Sauropodomorpha: Anchisauria), in Evolution and Palaeobiology of Early Sauropodomorph Dinosaurs, edited by P. M. Barrett, and D. J. Batten, pp. 9-55, Special Papers in Palaeontology, 77. 